\title{
Pheochromocytomas and pituitary adenomas in three patients with MAX exon deletions
}

\section{Dear Editor,}

Understanding of the genetic pathophysiology of endocrine tumors has advanced significantly and mutations in many causative genes are routinely sought. New syndromic associations among endocrine tumors have also been identified. These novel associations are important to recognize in order to direct screening appropriately. For instance, pheochromocytomas and pituitary adenomas can rarely occur together, usually due to germline $S D H x$ mutations (Dénes et al. 2015, Xekouki et al. 2015, Guerrero Pérez et al. 2016). Despite a multitude of known genetic risk factors, many unexplained cases of multiple endocrine tumors exist ( $\mathrm{O}^{\prime}$ Toole et al. 2015). New causative genes and other genomic mechanisms affecting previously identified genes need to be considered during diagnostic workup.

Copy number variations (CNV) are an important mechanism in inherited tumor genetics. CNV can affect large genetic regions or can be limited to deletions of single exons. Such exon-level intragenic deletions have been reported in connection with genes associated with isolated and syndromic endocrine neoplasia (Bayley et al. 2009, Ricketts et al. 2010, Zatelli et al. 2014). Identification of single exon deletions can be challenging. Techniques like multiplex ligation-dependent probe amplification (MLPA) and quantitative PCR are generally available and can identify CNV (e.g. deletions) of individual exons in cases where sequencing results are apparently normal.

Mutations in the MAX gene are associated with a risk of sporadic and hereditary pheochromocytoma and are also implicated in kidney tumors (Comino-Méndez et al. 2011, Burnichon et al. 2012). Recently a large deletion including the MAX promoter and exons 1 and 2 was identified in a family with bilateral pheochromocytoma and renal oncocytoma (Korpershoek et al. 2016). Using MLPA we studied six sporadic cases with multiple endocrine tumors that were negative on NGS panels and Sanger sequencing for known risk genes. Patients provided their consent and the study was approved by the Ethics Committee of the CHU de Liège. We identified three patients with pheochromocytomas and pituitary adenomas who had germline heterozygous MAX exon deletions.

Case 1 , a male patient, initially presented aged 32 years with a two-year history of gradually worsening headache, sweating and palpitations in association with severe hypertension. He had no known family history of endocrine tumors. Plasma epinephrine and norepinephrine and urinary norepinephrine, normetanephrine and vanillyl mandelic acid (VMA) levels were all markedly elevated. A $42 \times 36 \times 40 \mathrm{~mm}$ right adrenal lesion was identified on MRI and was histologically confirmed as a pheochromocytoma following a right-sided adrenalectomy (Fig. 1A). He was asymptomatic for many years. He presented again at the age of 49 with painful right-sided gynecomastia without galactorrhea; he denied headache and had no visual symptoms. His blood pressure was $133 / 80 \mathrm{mmHg}$ and his resting heart rate was 70 beats per minute. Hormonal testing revealed hyperprolactinemia (1372 IU/L; normal range: $85-325 \mathrm{IU} / \mathrm{L}$ ) and low free testosterone; other hormonal axes were normal. On MRI, he had an $8 \mathrm{~mm}$ pituitary microadenoma (Fig. 1B). A diagnosis of prolactinoma was made, and he responded well clinically and biochemically to cabergoline $0.5 \mathrm{mg} /$ week. Eighteen months later, the patient developed malaise, headache, sweating and palpitations with hypertension $(133 / 100 \mathrm{mmHg})$ and a resting heart rate of $80 / \mathrm{min}$. VMA, norepinephrine and normetanephrine were elevated. Abdominal MRI showed a 13-mm diameter nodular lesion close to the position of the previous right adrenalectomy. MIBG scintigraphy was negative. The lesion was resected and was confirmed histologically as a pheochromocytoma. Sequencing for RET, VHL, SDHx genes, CDKN1B, AIP and MEN1 was negative. During follow-up, the patient had a large $(20 \mathrm{~mm})$, hypo-echogenic thyroid nodule identified. Calcitonin levels were normal. Scintigraphy showed no uptake in the nodule, and fine-needle biopsy was negative 
but due to the history of endocrine tumors, he elected to undergo total thyroidectomy and the histology was benign.

Case 2, a female, presented originally aged 26 years with symptoms of acromegaly including acral enlargement. She had no family history of endocrine tumors. On investigation, she was found to have elevated growth hormone (GH) and insulin-like growth factor-1 (IGF1) secretion and an invasive pituitary macroadenoma was identified on MRI (Fig. 1F). As the tumor could not be completely surgically resected, the patient elected to receive a long-acting somatostatin analog in combination with cabergoline and later pegvisomant. She underwent radiotherapy and at long-term follow-up, she was clinically and biochemically controlled. At the age of 35, she presented with edema, shortness of breath and episodic severe perspiration. On examination, she was severely hypertensive and investigations revealed elevated plasma catecholamines and 24-h urinary normetanephrine levels. Imaging revealed mass lesions in both adrenal glands consistent with pheochromocytomas (Fig. 1G), which were confirmed histologically following bilateral adrenalectomy. Further investigation for other tumor sites revealed a single thyroid nodule $(9 \mathrm{~mm})$. Calcitonin levels were normal. The thyroid lesion was determined to be a follicular variant of papillary thyroid cancer. She underwent total thyroidectomy, with clear lymph node and neck dissection. Given the history of tumors in three endocrine glands she underwent genetic testing for pheochromocytoma-paraganglioma, pituitary and MEN syndrome genes but no pathological variants were found.

Case 3 was a male patient with no family history of endocrine tumors who presented at 16 years of age with acral enlargement suggestive of acromegaly. He had elevated GH levels and a pituitary macroadenoma was identified. He underwent a gross total resection of the pituitary adenoma and received postoperative radiotherapy, which led to clinical and biochemical control of acromegaly and hypopituitarism that was substituted. At the age of 22 , he was noted to be markedly hypertensive during follow-up, and upon investigation, elevated VMA levels and urinary noradrenaline were noted. Imaging revealed an enlargement of the left adrenal gland, which was confirmed as a pheochromocytoma following surgical resection. However, the patient remained hypertensive with elevated urinary catecholamines post-operatively and at second surgery a residual pheochromocytoma was identified. He remained symptom free but on antihypertensive medication. Eleven years after his initial pheochromocytoma surgery, he developed elevated levels of VMA and plasma norepinephrine. Thoracic and abdominal CT revealed a $35 \times 40 \mathrm{~mm}$ lesion on the right adrenal and a $42 \times 30 \mathrm{~mm}$ lesion at the surgical site of the left pheochromocytoma (Fig. 2E). He underwent adrenalectomy, which confirmed the presence of bilateral pheochromocytomas. Investigations for pheochromocytoma-paraganglioma, pituitary adenoma and MEN syndrome risk factor genes were negative. During follow-up, the patient developed metastatic deposits from the pheochromocytoma and he died due to disease progression.

As sequencing of known risk factor genes for pheochromocytoma, pituitary adenomas and MEN syndromes was normal (including $M A X$ ), we undertook MLPA studies using available kits for SDHx, VHL, MEN1, $C D K N 1 B, A I P$ and MAX (details available on request). Two of the patients demonstrated germinal heterozygous deletions of single exons of MAX: exon 3 (Case 1; Fig. 1D) and exon 4 (Case 3; Fig. 2G). Case 2 had a germinal heterozygous deletion of MAX exons 1-3 and intron 3 (Fig. 2A). SNPs on the probe hybridization sites were excluded by sequencing.

Immunohistochemical staining of the pheochromocytomas from all three patients demonstrated loss of nuclear staining for MAX (Figs $1 \mathrm{C}$ and $\mathrm{H}$ and $2 \mathrm{H}$ ). As shown in Fig. 2B and C, genetic analysis of tumor DNA from the left- and right-sided pheochromocytomas of Case 2 demonstrated that there was a homozygous loss of MAX exons 1-3 and intron 3 indicating a second genetic 'hit' at the normal allele and loss of heterozygosity. In contrast, DNA analysis of the thyroid carcinoma showed the MAX exon 1-3 deletion was present only in the heterozygous state. In support of this, the thyroid carcinoma retained positive nuclear staining for MAX, indicating that the nonmutated allele was not lost (Fig. 2D). Pituitary tumor tissue from Case 3 was not available for immunohistochemical analysis. Family screening studies identified the inheritance of the same $M A X$ exon 3 deletion in the asymptomatic 30-year-old son of Case 1.

Co-existence of pituitary adenomas and pheochromocytomas-paragangliomas is rare (termed 3PA syndrome by Xekouki et al.) and is usually associated with $S D H x$ mutations, although genetically negative cases exist (O'Toole et al. 2015, Xekouki et al. 2015, Guerrero Pérez et al. 2016). We have shown that MAX exon/intragenic deletions might also explain this pituitary adenoma-pheochromocytoma association. MAX MLPA should be considered in such syndromic cases and in pheochromocytoma cases where a genetic cause is sought, as individual MAX exon deletions are generally 

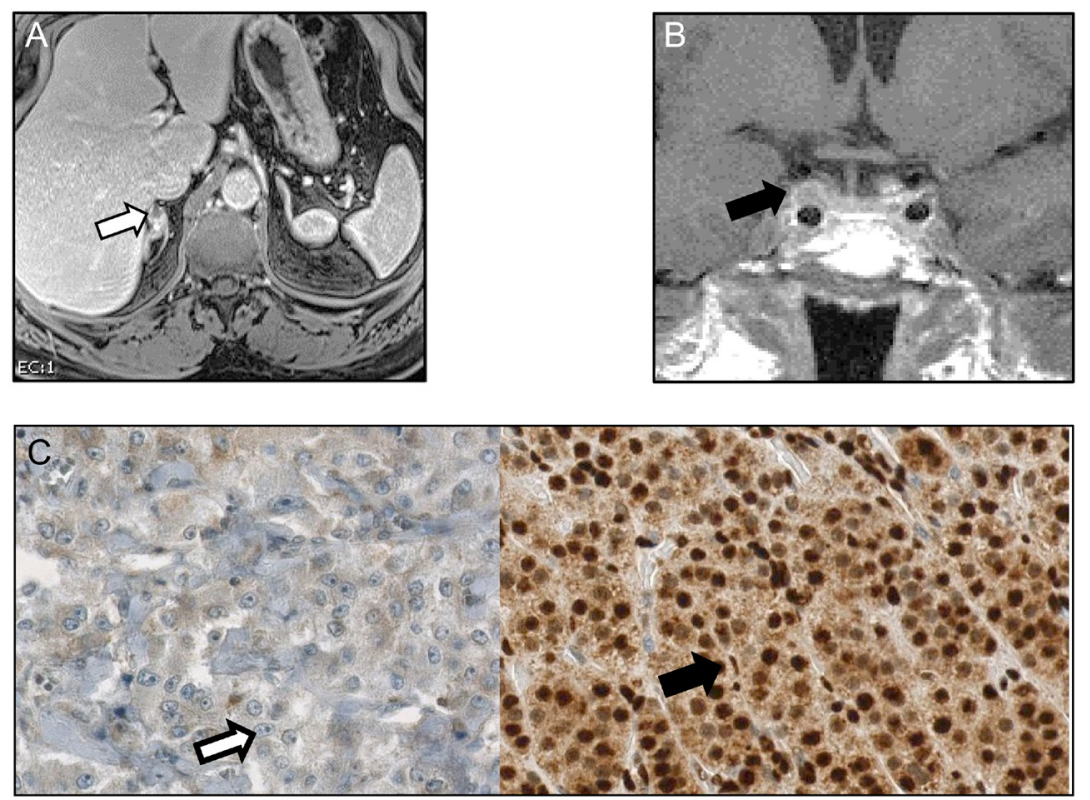

$\mathrm{D}$

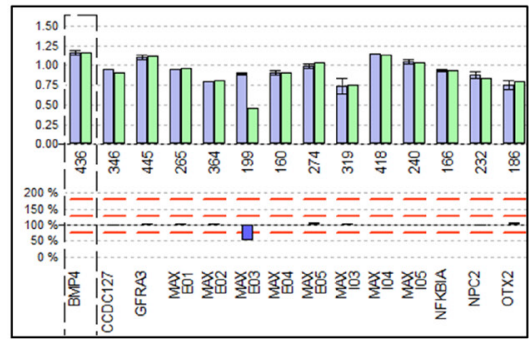

$\mathrm{F}$

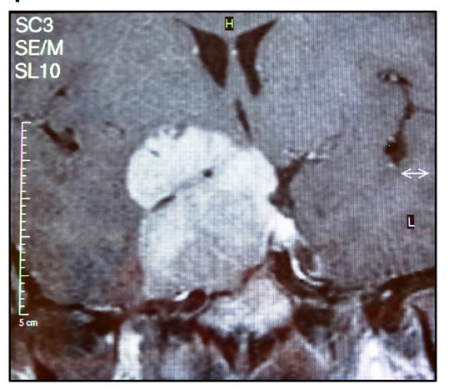

E

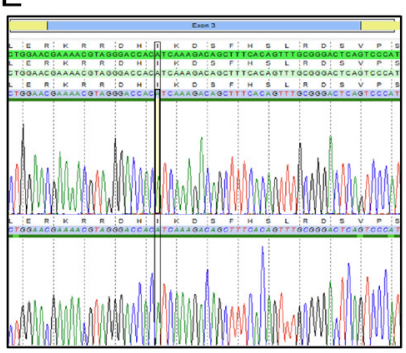

G

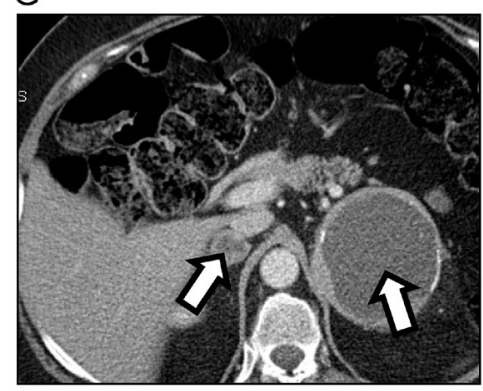

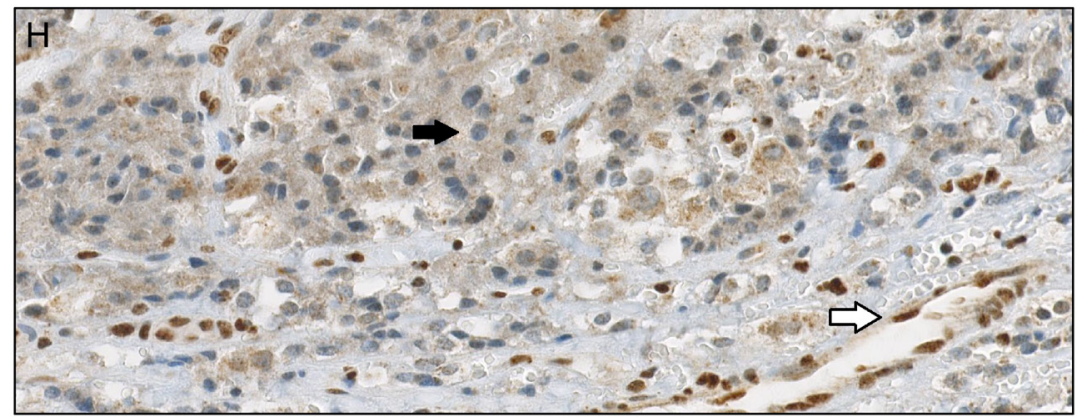

\section{Figure 1}

Panel $A$ is a gadolinium-enhanced T1 sequence MRI showing a $15-\mathrm{mm}$ diameter lesion

(pheochromocytoma) in the right adrenal in Case 1. Panel $B$ is a gadolinium enhanced T1 sequence MRI showing a non-enhancing pituitary microadenoma (prolactinoma; arrow). Panel C shows the results of immunohistochemistry for MAX (C-17; sc-197, Santa Cruz Biotechnology) in the recurrent pheochromocytoma in Case 1. The difference between absent MAX nuclear staining on the left side of the image (blue; open arrow) and the intact MAX nuclear staining (dark brown; filled arrow) in the patient's normal adrenal tissue on the right side of the image can be readily seen. Panel $D$ shows the MLPA results for leukocyte derived DNA demonstrating a germinal deletion of exon 3 of the $M A X$ gene in Case 1. Panel $\mathrm{E}$ shows the electropherogram of germline DNA from Case 1 that demonstrates a normal sequence result despite a discrete exon 3 deletion. Panel $\mathrm{F}$ demonstrates a coronal MRI image of the somatotropinoma at diagnosis in Case 2 and Panel $\mathrm{G}$ shows an abdominal CT scan of Case 2 with bilateral pheochromocytomas at diagnosis (arrows). Panel $\mathrm{H}$ shows the immunohistochemical staining of a pheochromocytoma from Case 2 demonstrating negative nuclear MAX stain (blue; filled arrow), as compared with intact nuclear staining in adjacent normal tissue (brown; open arrow). 

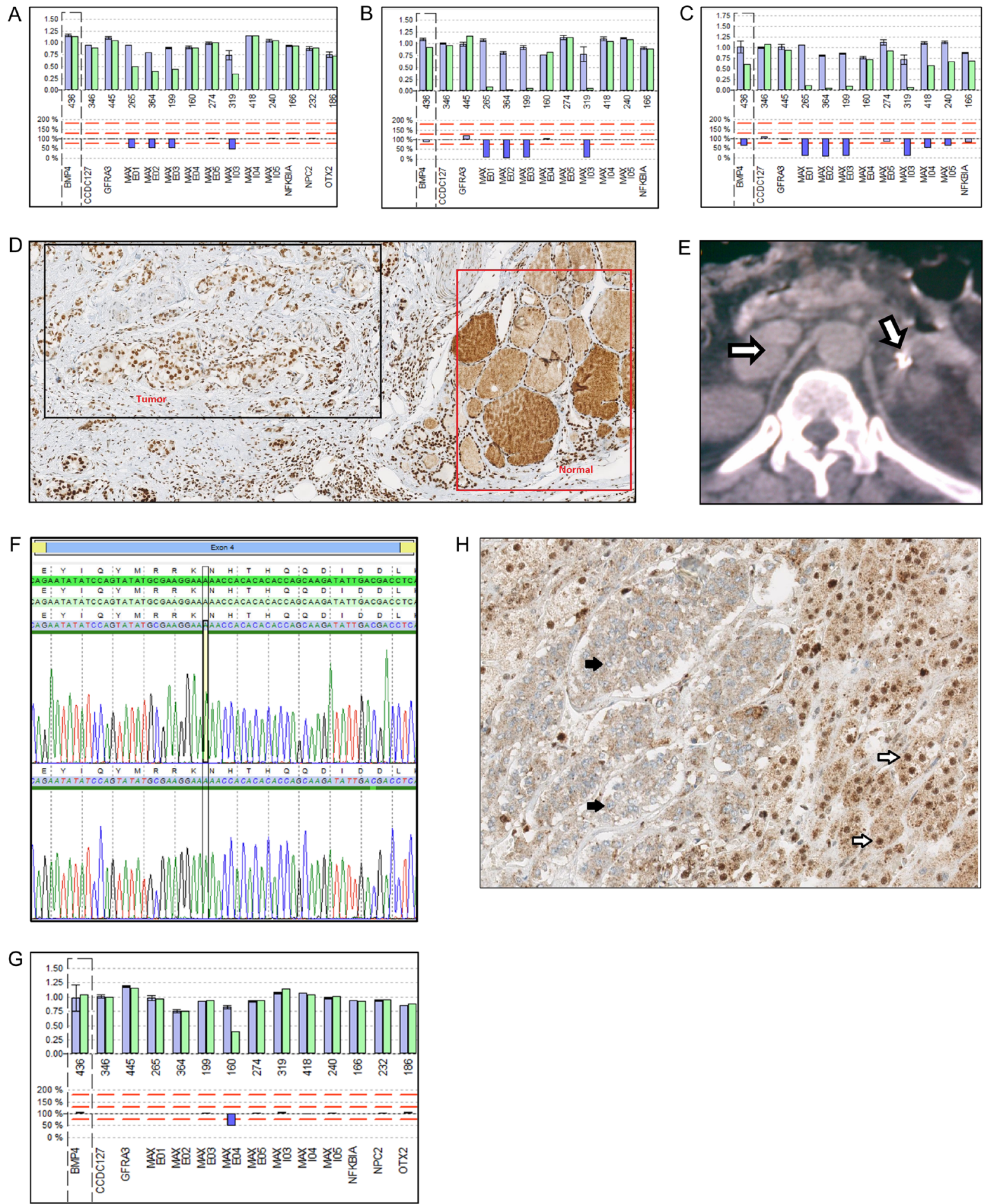

Figure 2

Panel $A$ is the MLPA kit result for Case 2, with a deletion of exons 1-3 and intron 3 of MAX present in the germline DNA. Homozygous deletion of MAX exon 1 to intron 3 in DNA from both right (Panel B) and left (Panel C) pheochromocytomas is shown, indicating a 'second hit' in both tumors. It should be noted that the second hit on the right pheochromocytoma partially involved MAX introns 4 and 5 . Panel D shows intact MAX staining in both normal thyroid tissue and thyroid carcinoma from patient 2, suggesting that the carcinoma was not linked to the MAX deletion. Panel E shows the CT image of the bilateral pheochromocytomas in Case 3 (one recurrent and one de novo). Panel $\mathrm{F}$ is the electropherogram from MAX gene sequencing that illustrates a normal germline DNA sequence at exon 4, despite the demonstration of a discrete exon 4 deletion on the MLPA result in Panel G. Panel $\mathrm{H}$ is an image showing the immunohistochemical staining for MAX in one pheochromocytoma from Case 3, with absent nuclear MAX staining shown in cells on the left side of the image (blue; filled arrows); normal MAX nuclear staining (dark brown) was present in adjacent normal adrenal tissue (open arrows). 
not identifiable on sequencing (Figs $1 \mathrm{E}$ and 2F). Including a recently reported case from Roszko et al. with a MAX mutation (Roszko et al. 2017), pituitary adenomas have been described in four cases of germline MAX exonic deletions or mutations; they may either precede or follow the appearance of a pheochromocytoma and can have variable secretory characteristics (acromegaly $(n=2)$ or prolactinoma $(n=2))$. The two somatotropinomas were early-onset, large, invasive tumors at diagnosis. Although a definitive tissue-based relationship between pituitary adenomas and MAX exon deletions/mutations remains to be shown, screening studies of pheochromocytoma patients and relatives should take into account the potential for the occurrence of pituitary pathology. In addition, exon deletions in MAX can be inherited. MLPA or other techniques to identify discrete MAX exon deletions should be considered in individuals and kindreds with pheochromocytoma that are negative following comprehensive sequencing and NGS panel testing.

\section{Adrian F Daly1,* \\ Emilie Castermans ${ }^{2, *}$ \\ Lindsey Oudijk ${ }^{3}$ \\ Mirtha A Guitelman ${ }^{4}$ \\ Pablo Beckers ${ }^{5}$ \\ Iulia Potorac ${ }^{1}$ \\ Sebastian J C M M Neggers 6 \\ Nathalie Sacre ${ }^{2}$ \\ Aart-Jan van der Lely ${ }^{6}$ \\ Vincent Bours ${ }^{2}$ \\ Wouter W de Herder ${ }^{6}$ \\ Albert Beckers ${ }^{1}$}

${ }^{1}$ Department of Endocrinology, Centre Hospitalier Universitaire de Liège, Liège Université, Liège, Belgium

${ }^{2}$ Department of Human Genetics, Centre Hospitalier Universitaire de Liège, Liège Université, Liège, Belgium

${ }^{3}$ Department of Pathology, Erasmus Medical Center, Rotterdam, The Netherlands

${ }^{4}$ Division of Endocrinology, Hospital Carlos G. Durand, Buenos Aires, Argentina

${ }^{5}$ Department of Clinical Chemistry, Centre Hospitalier Universitaire de Liège, Liège Université, Liège, Belgium ${ }^{6}$ Department of Endocrinology, Erasmus Medical Center, Rotterdam, The Netherlands

Correspondence should be addressed to A Beckers: albert.beckers@chu.ulg.ac.be

*(A F Daly and E Castermans contributed equally to this work)

\section{Declaration of interest}

The authors declare that there is no conflict of interest that could be perceived as prejudicing the impartiality of this article.

\section{Funding}

The work was supported by a Fonds d'investissement pour la Recherche (FIRS) grant from the Centre Hospitalier Universitaire de Liège, and a grant from the JABBS Foundation, UK.

\section{Acknowledgements}

The authors would like to that Dr E Korpershoek for discussions on MAX immunohistochemistry.

\section{References}

Bayley J-P, Weiss MM, Grimbergen A, van Brussel BTJ, Hes FJ, Jansen JC, Verhoef S, Devilee P, Corssmit EP \& Vriends AHJT 2009 Molecular characterization of novel germline deletions affecting SDHD and SDHC in pheochromocytoma and paraganglioma patients. Endocrine-Related Cancer 16 929-937. (https://doi. org/10.1677/ERC-09-0084)

Burnichon N, Cascon A, Schiavi F, Morales NP, Comino-Mendez I, Abermil N, Inglada-Perez L, de Cubas AA, Amar L, Barontini M, et al. 2012 MAX mutations cause hereditary and sporadic pheochromocytoma and paraganglioma. Clinical Cancer Research 18 2828-2837. (https://doi.org/10.1158/1078-0432.CCR-12-0160)

Comino-Méndez I, Gracia-Aznárez FJ, Schiavi F, Landa I, LeandroGarcía LJ, Letón R, Honrado E, Ramos-Medina R, Caronia D, Pita G, et al. 2011 Exome sequencing identifies MAX mutations as a cause of hereditary pheochromocytoma. Nature Genetics 43 663-667. (https://doi.org/10.1038/ng.861)

Dénes J, Swords F, Rattenberry E, Stals K, Owens M, Cranston T, Xekouki P, Moran L, Kumar A, Wassif C, et al. 2015 Heterogeneous genetic background of the association of pheochromocytoma/ paraganglioma and pituitary adenoma: results from a large patient cohort. Journal of Clinical Endocrinology and Metabolism 100 E531-E541. (https://doi.org/10.1210/jc.2014-3399)

Guerrero Pérez F, Lisbona Gil A, Robledo M, Iglesias P \& Villabona Artero C 2016 Pituitary adenoma associated with pheochromocytoma/paraganglioma: a new form of multiple endocrine neoplasia. Endocrinología Y Nutrición 63 506-508. (https:// doi.org/10.1016/J.ENDOEN.2016.10.012)

Korpershoek E, Koffy D, Eussen BH, Oudijk L, Papathomas TG, van Nederveen FH, Belt EJT, Franssen GJH, Restuccia DFJ, Krol NMG, et al. 2016 Complex MAX rearrangement in a family with malignant pheochromocytoma, renal oncocytoma, and erythrocytosis. Journal of Clinical Endocrinology and Metabolism 101 453-460. (https://doi. org/10.1210/jc.2015-2592)

O’Toole SM, Dénes J, Robledo M, Stratakis CA \& Korbonits M 201515 years of paraganglioma: the association of pituitary adenomas and phaeochromocytomas or paragangliomas. Endocrine-Related Cancer 22 T105-T122. (https://doi.org/10.1530/ERC-15-0241)

Ricketts CJ, Forman JR, Rattenberry E, Bradshaw N, Lalloo F, Izatt L, Cole TR, Armstrong R, Kumar VKA, Morrison PJ, et al. 2010 Tumor risks and genotype-phenotype-proteotype analysis in 358 patients with germline mutations in SDHB and SDHD. Human Mutation 31 41-51. (https://doi.org/10.1002/humu.21136)

Roszko KL, Blouch E, Blake M, Powers JF, Tischler AS, Hodin R, Sadow P \& Lawson EA 2017 Case report of a prolactinoma in a patient with a novel MAX mutation and bilateral pheochromocytomas. Journal of (c) 2018 Society for Endocrinology Published by Bioscientifica Ltd. Printed in Great Britain 
the Endocrine Society 1 1401-1407. (https://doi.org/10.1210/js.201700135)

Xekouki P, Szarek E, Bullova P, Giubellino A, Quezado M, Mastroyannis SA, Mastorakos P, Wassif CA, Raygada M, Rentia N, et al. 2015Pituitary adenoma with paraganglioma/ pheochromocytoma (3PAs) and succinate dehydrogenase defects in humans and mice. Journal of Clinical Endocrinology and
Metabolism 100 E710-E719. (https://doi.org/10.1210/jc.20144297)

Zatelli MC, Tagliati F, Di Ruvo M, Castermans E, Cavazzini L, Daly AF, Ambrosio MR, Beckers A \& degli Uberti E 2014 Deletion of exons 1-3 of the MEN1 gene in a large Italian family causes the loss of menin expression. Familial Cancer 13 273-280. (https://doi. org/10.1007/s10689-014-9702-y)

Received in final form 9 March 2018

Accepted 13 March 2018

Accepted Preprint published online 13 March 2018 (c) 2018 Society for Endocrinology Published by Bioscientifica Ltd. Printed in Great Britain 